\title{
An Approach to the Concept of Soft Fuzzy Proximity
}

\author{
Vildan Çetkin, ${ }^{1}$ Alexander P. Šostak, ${ }^{2}$ and Halis Aygün ${ }^{1}$ \\ ${ }^{1}$ Department of Mathematics, University of Kocaeli, Umuttepe Campus, 41380 Kocaeli, Turkey \\ ${ }^{2}$ Department of Mathematics, University of Latvia, Riga 1002, Latvia
}

Correspondence should be addressed to Vildan Çetkin; vcetkin@gmail.com

Received 2 August 2013; Accepted 9 December 2013; Published 26 March 2014

Academic Editor: Gerd Teschke

Copyright (C) 2014 Vildan Çetkin et al. This is an open access article distributed under the Creative Commons Attribution License, which permits unrestricted use, distribution, and reproduction in any medium, provided the original work is properly cited.

\begin{abstract}
The purpose of this paper is to introduce the concept of soft fuzzy proximity. Firstly, we give the definitions of soft fuzzy proximity and Katsaras soft fuzzy proximity, and also we investigate the relations between the soft fuzzy proximity and slightly modified version of Katsaras soft fuzzy proximity. Secondly, we induce a soft fuzzy topology from a given soft fuzzy proximity by using soft fuzzy closure operator. Then, we obtain the initial soft fuzzy proximity from a given family of soft fuzzy proximities. So, we describe products in the category of soft fuzzy proximities. Finally, we show that a family of all soft fuzzy proximities on a given set constitutes a complete lattice.
\end{abstract}

\section{Introduction}

In 1999, Molodtsov [1] proposed a completely new concept called soft set theory to model uncertainty, which associates a set with a set of parameters. Later, Maji et al. [2] introduced the concept of fuzzy soft set which combines fuzzy sets and soft sets. Soft set and fuzzy soft set theories have a rich potential for applications in several directions. The algebraic structure of soft set and fuzzy soft set theories dealing with uncertainties has been studied by some authors [3-10]. The topological structure of fuzzy soft sets based on the sense of Šostak [11] has also been studied by Aygünoğlu et al. [12]. They proved that the category of fuzzy soft topological spaces is a topological category over $\mathrm{SET}^{3}$.

There are known some different approaches to the concept of a fuzzy proximity in the literature. The first and the most advanced one was developed on the whole by Katsaras $[13,14]$, the second due to Artico and Moresco $[15,16]$. Although these approaches proceed from different starting points, both of them are consistent with Chang (or Lowen) fuzzy topologies. Markin and Šostak [17] introduced the different concept of a fuzzy proximity which is consistent with the notion of a fuzzy topology as it is defined in [11]. They considered basic properties of these fuzzy proximities, described how a fuzzy proximity generates a fuzzy topology (in the sense of [11]), and discussed the interrelations between their approaches and each of the two abovementioned approaches to the concept of a fuzzy proximity. The notion of an $L$ fuzzy preproximity spaces where $L$ is a strictly two-sided, commutative quantale lattice having a strong negation was introduced by Kim and Min [18] from a somewhat different point from that in [17]. Double fuzzy preproximity was introduced and studied by Zahran et al. [19]. Cetkin and Aygün [20] gave the definition of a lattice valued double fuzzy preproximity spaces as an extension of [18] and studied some of its structural properties.

Despite the existence of all these approaches to the concept of fuzzy proximity, we decide to generalize more natural definition of fuzzy proximity to the soft theory. In this paper, we give an approach to the concept of soft fuzzy proximity which is the extension of the fuzzy proximities studied in [17] to the soft theory. In the case of the parameter sets $E$ and $K$ that are both one-pointed sets, we obtain the results given in [17]. Firstly, we define soft fuzzy proximity space and Katsaras soft fuzzy proximity space and also investigate the relations between these spaces. Secondly, we generate a soft fuzzy topology from a given soft fuzzy proximity by using the closure operator. Thirdly, we prove the existence of the initial soft fuzzy proximity to describe products in the category SFP of soft fuzzy proximity spaces and soft proximally continuous maps. Finally, we show that a family of all soft fuzzy proximities on a given set is a complete lattice. 


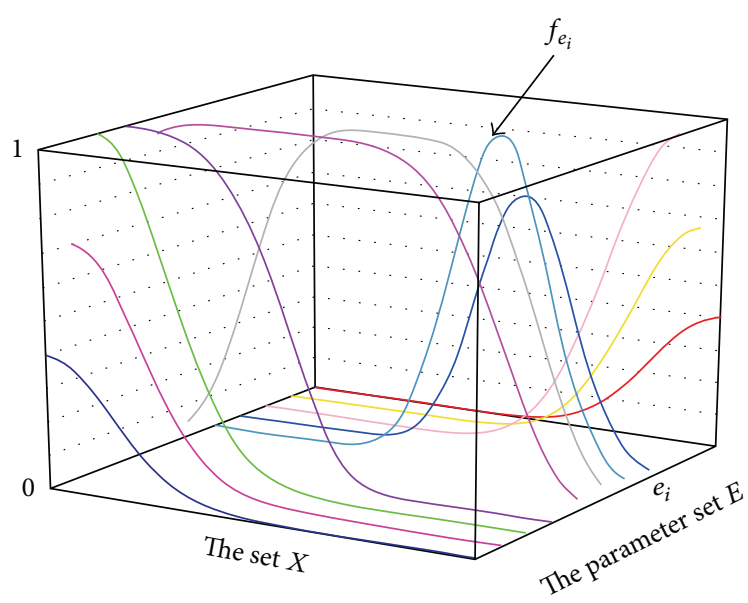

Figure 1: A fuzzy soft set $f$.

\section{Preliminaries}

Throughout this paper, $X$ refers to an initial universe, $E$ is the set of all parameters for $X$, and $I^{X}$ is the set of all fuzzy sets on $X$ (where $I=[0,1])$. For $\alpha \in I, \bar{\alpha}(x)=\alpha$ for all $x \in X$. A fuzzy point in $I^{X}$ is a fuzzy set $x^{\alpha}$, where $\alpha \in(0,1]$, such that $x^{\alpha}(y)=\alpha$ when $y=x$ and $x^{\alpha}(y)=0$ otherwise. A family of fuzzy points is denoted by $\operatorname{Pt}(X)$.

Definition 1 (see [21]). $f$ is called a fuzzy soft set on $X$, where $f$ is a mapping from $E$ into $I^{X}$; that is, $f_{e} \triangleq f(e)$ is a fuzzy set on $X$, for each $e \in E$. The family of all $I$-fuzzy soft sets on $X$ is denoted by $\left(I^{X}\right)^{E}$ see (Figure 1).

Definition 2 (see [21]). Let $f$ and $g$ be two fuzzy soft sets on $X$; then

(1) we say that $f$ is a fuzzy soft subset of $g$ and write $f \sqsubseteq g$ if $f_{e} \leq g_{e}$, for each $e \in E$. $f$ and $g$ are called equal if $f \sqsubseteq g$ and $g \sqsubseteq f$;

(2) the union of $f$ and $g$ is a fuzzy soft set $h=f \sqcup g$, where $h_{e}=f_{e} \vee g_{e}$, for each $e \in E$;

(3) the intersection of $f$ and $g$ on $X$ is a fuzzy soft set $h=f \sqcap g$, where $h_{e}=f_{e} \wedge g_{e}$, for each $e \in E ;$

(4) the complement of a fuzzy soft set $f$ is denoted by $f^{\prime}$, where $f^{\prime}: E \rightarrow I^{X}$ is a mapping given by $f_{e}^{\prime}=\overline{1}-f_{e}$, for each $e \in E$;

(5) $f$ is called a null fuzzy soft set and denoted by $0_{X}$, if $f_{e}(x)=0$, for each $e \in E, x \in X$;

(6) $f$ is called an absolute fuzzy soft set and denoted by $1_{X}$, if $f_{e}(x)=1$, for each $e \in E, x \in X$.

Definition 3. A fuzzy soft point $P$ is a fuzzy soft set, where $P: E \rightarrow P t(X) ; P(e)=x^{\alpha}$ for all $e \in E$. In other words, a fuzzy soft point can be considered as a collection of fuzzy points with respect to the parameters. A fuzzy soft point $P$ is said to belong to a fuzzy soft set $g$, denoted by $P \in g$ if and only if $P \sqsubseteq g$ or equivalently $\alpha \leq g_{e}(x)$, for each $e \in E$.
Definition 4 (see [4]). Let $\varphi: X_{1} \rightarrow X_{2}$ and $\psi: E_{1} \rightarrow E_{2}$ be two functions, where $E_{1}$ and $E_{2}$ are parameter sets for the crisp sets $X_{1}$ and $X_{2}$, respectively. Then the pair $\varphi_{\psi}$ is called a fuzzy soft mapping from $X_{1}$ to $X_{2}$. Let $f$ and $g$ be two fuzzy soft sets on $X_{1}$ and $X_{2}$, respectively.

(1) The image of $f$ under the fuzzy soft mapping $\varphi_{\psi}$, denoted by $\varphi_{\psi}(f)$, is the fuzzy soft set on $X_{2}$ defined by

$$
\begin{aligned}
& \varphi_{\psi}(f)_{k}(y) \\
& = \begin{cases}\bigvee_{\varphi(x)=y} \bigvee_{\psi(a)=k} f_{a}(x), & \text { if } x \in \varphi^{-1}(y), a \in \psi^{-1}(k), \\
0, & \text { otherwise. }\end{cases}
\end{aligned}
$$

$$
\forall k \in E_{2}, \quad y \in X_{2} \text {. }
$$

(2) The preimage of $g$ under the fuzzy soft mapping $\varphi_{\psi}$, denoted by $\varphi_{\psi}^{-1}(g)$, is the fuzzy soft set on $X_{1}$ defined by

$\varphi_{\psi}^{-1}(g)_{e}(x)=g_{\psi(e)}(\varphi(x)), \quad \forall e \in E_{1}, x \in X_{1}$.

If $\varphi$ and $\psi$ are injective (surjective), then $\varphi_{\psi}$ is said to be injective (surjective).

(3) Let $\varphi_{\psi}$ be a fuzzy soft mapping from $X_{1}$ to $X_{2}$ and let $\varphi_{\psi^{*}}^{*}$ be a fuzzy soft mapping from $X_{2}$ to $X_{3}$. Then the composition of these mappings from $X_{1}$ to $X_{3}$ is defined as follows: $\varphi_{\psi} \circ \varphi_{\psi^{*}}^{*}=\left(\varphi \circ \varphi^{*}\right)_{\psi \circ \psi^{*}}$, where $\psi: E_{1} \rightarrow E_{2}$ and $\psi^{*}: E_{2} \rightarrow E_{3}$.

(4) The image of the fuzzy soft point $P$ under the fuzzy soft mapping $\varphi_{\psi}$ is defined as follows:

$$
\varphi_{\psi}(P)_{k}=\varphi(P)_{\psi(k)}=\varphi\left(x^{\alpha}\right)=\varphi(x)^{\alpha}, \quad \text { for each } k \in E_{2} \text {. }
$$

For more details about fuzzy soft sets and fuzzy soft mappings, please refer to $[2,4,12,21-23]$.

The second parameter set belonging to the context of our work is denoted by $K$, which is the parameter set for the fuzzy soft topological structures.

So throughout this study, let $E$ and $K$ be arbitrary nonempty sets viewed on the sets of parameters.

Definition 5 (see [12]). A mapping $\tau: K \rightarrow I^{\left(I^{X}\right)^{E}}$ is called an $(E, K)$-soft fuzzy topology on $X$ if it satisfies the following conditions for each $k \in K$ :

(O1) $\tau_{k}\left(0_{X}\right)=\tau_{k}\left(1_{X}\right)=1$;

(O2) $\tau_{k}(f \sqcap g) \geq \tau_{k}(f) \wedge \tau_{k}(g)$, for all $f, g \in\left(I^{X}\right)^{E}$;

(O3) $\tau_{k}\left(\bigsqcup_{i \in \Gamma} f_{i}\right) \geq \bigwedge_{i \in \Gamma} \tau_{k}\left(f_{i}\right)$, for all $f_{i} \in\left(I^{X}\right)^{E}, i \in \Delta$.

Then the pair $(X, \tau)$ is called an $(E, K)$-soft fuzzy topological space. The value $\tau_{k}(f)$ is interpreted as the degree of openness of a fuzzy soft set $f$ with respect to parameter $k \in K$. 
Let $\tau^{1}$ and $\tau^{2}$ be $(E, K)$-soft fuzzy topologies on $X$. We say that $\tau^{1}$ is finer than $\tau^{2}$ ( $\tau^{2}$ is coarser than $\tau^{1}$ ), denoted by $\tau^{2} \leq \tau^{1}$, if $\tau_{k}^{2}(f) \leq \tau_{k}^{1}(f)$ for each $k \in K, f \in\left(I^{X}\right)^{E}$.

Example 6 (see [12]). Let $\mathscr{T}$ be a fuzzy topology on $X$ in Sostak's sense; that is, $\mathscr{T}$ is a mapping from $I^{X}$ to $I$. Take $K=E=I$ and define $\overline{\mathscr{T}}: E \rightarrow I^{X}$ as $\overline{\mathscr{T}}(e) \triangleq\{\mu: \mathscr{T}(\mu) \geq e\}$ which is levelwise fuzzy topology of $\mathscr{T}$ in Chang's sense, for each $e \in I$. However, it is well known that each Chang's fuzzy topology can be considered as Šostak fuzzy topology by using fuzzifying method. Hence, $\mathscr{T}(e)$ satisfies $(\mathrm{O} 1),(\mathrm{O} 2)$, and (O3).

According to this definition and by using the decomposition theorem of fuzzy sets [24], if we know the resulting soft fuzzy topology, then we can find the first fuzzy topology. Therefore, we can say that a fuzzy topology can be uniquely represented as a soft fuzzy topology.

Example 7. Let $E$ be a parameter set, let $K=\mathbb{N}$ be the set of natural numbers, and let $\tau: K \rightarrow I^{\left(^{X}\right)^{E}}$ be defined as follows: for each $k \in K$,

$$
\tau_{k}(f)= \begin{cases}1, & \text { if } f=0_{X}, 1_{X} \\ \frac{1}{k}, & \text { otherwise. }\end{cases}
$$

It is easy to testify that $\tau$ is a soft fuzzy topology on $X$.

Definition 8 (see [12]). Let $\left(X_{1}, \tau^{1}\right)$ be an $\left(E_{1}, K_{1}\right)$-soft fuzzy topological space and let $\left(X_{2}, \tau^{2}\right)$ be an $\left(E_{2}, K_{2}\right)$-soft fuzzy topological space. Let $\varphi: X_{1} \rightarrow X_{2}, \psi: E_{1} \rightarrow E_{2}$ and $\eta: K_{1} \rightarrow K_{2}$ be functions. Then the mapping $\varphi_{\psi, \eta}$ from $\left(X_{1}, \tau^{1}\right)$ into $\left(X_{2}, \tau^{2}\right)$ is called a soft fuzzy continuous map if $\tau_{k}^{1}\left(\varphi_{\psi}^{-1}(g)\right) \geq \tau_{\eta(k)}^{2}(g)$ for all $g \in\left(I^{X_{2}}\right)^{E_{2}}, k \in K_{1}$.

The category of soft fuzzy topological spaces and soft fuzzy continuous mappings is denoted by SFTOP.

Definition 9 (see [25]). A map $\mathscr{C}: K \times\left(I^{X}\right)^{E} \times I_{0} \rightarrow\left(I^{X}\right)^{E}$ is called an $(E, K)$-soft fuzzy closure operator on $X$ if and only if $\mathscr{C}$ satisfies the following conditions, for each $k \in K, \alpha \in I_{0}$ (where $I_{0}=I \backslash\{0\}$ ) and $f, g \in\left(I^{X}\right)^{E}$ :
(C1) $\mathscr{C}\left(k, 0_{X}, \alpha\right)=0_{X}$;
(C2) $\mathscr{C}(k, f, \alpha) \sqsupseteq f$;
(C3) if $f \sqsubseteq g$, then $\mathscr{C}(k, f, \alpha) \sqsubseteq \mathscr{C}(k, g, \alpha)$;
(C4) if $\alpha \leq \beta$, then $\mathscr{C}(k, f, \alpha) \sqsubseteq \mathscr{C}(k, f, \beta)$;
(C5) $\mathscr{C}(k, f \sqcup g, \alpha)=\mathscr{C}(k, f, \alpha) \sqcup \mathscr{C}(k, g, \alpha)$.

The pair $(X, \mathscr{C})$ is called an $(E, K)$-soft fuzzy closure space. An $(E, K)$-soft fuzzy closure space $(X, \mathscr{C})$ is called topological if it provides

$$
\begin{array}{r}
\mathscr{C}(k, \mathscr{C}(k, f, \alpha), \alpha)=\mathscr{C}(k, f, \alpha), \quad \text { for each } k \in K, \\
\alpha \in I_{0}, f \in\left(I^{X}\right)^{E} .
\end{array}
$$

Definition 10 (see [25]). Let $\left(X_{1}, \mathscr{C}^{1}\right)$ be an $\left(E_{1}, K_{1}\right)$-soft fuzzy closure space and, $\left(X_{2}, \mathscr{C}^{2}\right)$ be an $\left(E_{2}, K_{2}\right)$-soft fuzzy closure space. Let $\varphi: X_{1} \rightarrow X_{2}, \psi: E_{1} \rightarrow E_{2}$ and $\eta: K_{1} \rightarrow K_{2}$ be functions. Then, a map $\varphi_{\psi, \eta}$ from $X_{1}$ to $X_{2}$ is called a soft fuzzy $\mathscr{C}$-map if

$$
\begin{gathered}
\varphi_{\psi}\left(\mathscr{C}^{1}(k, f, \alpha)\right) \sqsubseteq \mathscr{C}^{2}\left(\eta(k), \varphi_{\psi}(f), \alpha\right), \\
\text { for each } k \in K_{1}, f \in\left(I^{X_{1}}\right)^{E_{1}}, \alpha \in I_{0} .
\end{gathered}
$$

\section{Soft Fuzzy Proximity}

In this section, we define soft fuzzy proximity structures in the sense of Šostak and Markin and Katsaras, respectively. Then we investigate their relations from the categorical point of view.

Definition 11. A mapping $\delta: K \rightarrow I^{\left(I^{X}\right)^{E} \times\left(I^{X}\right)^{E}}$ is called an $(E, K)$-soft fuzzy proximity if it satisfies the following axioms for each $k \in K$ :

$$
\begin{aligned}
& \text { (P1) } \delta_{k}\left(0_{X}, 1_{X}\right)=0 \text {; } \\
& \text { (P2) } \delta_{k}(f, g)=\delta_{k}(g, f) \text {, for all } f, g \in\left(I^{X}\right)^{E} \text {; } \\
& \text { (P3) } \delta_{k}(f, g \sqcup h)=\delta_{k}(f, g) \vee \delta_{k}(f, h) \text {, for all } f, g, h \in\left(I^{X}\right)^{E} \text {; } \\
& \text { (P4) } \delta_{k}(f, g) \geq \sup _{x \in X} \sup _{e \in E}(f \sqcap g)_{e}(x) \text {; } \\
& \text { (P5) } \delta_{k}(f, g) \geq \inf \left\{\delta_{k}(f, h) \vee \delta_{k}\left(g, h^{\prime}\right): h \in\left(I^{X}\right)^{E}\right\} .
\end{aligned}
$$

A pair $(X, \delta)$ where $X$ is a set and $\delta$ is an $(E, K)$-soft fuzzy proximity on it is called an $(E, K)$-soft fuzzy proximity space. Also, we call $\delta_{k}(f, g)$ a gradation of nearness between the fuzzy soft sets $f$ and $g$ according to the parameter $k \in K$. Here, for each $k \in K, \delta_{k}$ is a mapping from $\left(I^{X}\right)^{E} \times\left(I^{X}\right)^{E}$ into I.

Remark 12. It is easy to notice that if $\delta$ is an $(E, K)$-soft fuzzy proximity on $X$, then $\delta_{k}\left(f, 0_{X}\right)=0$ for each $f \in\left(I^{X}\right)^{E}, k \in K$. If $f, g, h \in\left(I^{X}\right)^{E}$ and $g \sqsubseteq h$, then $\delta_{k}(f, g) \leq \delta_{k}(f, h)$ for all $k \in K$.

Definition 13. Let $\left(X_{1}, \delta^{1}\right)$ be an $\left(E_{1}, K_{1}\right)$-soft fuzzy proximity space and let $\left(X_{2}, \delta^{2}\right)$ be an $\left(E_{2}, K_{2}\right)$-soft fuzzy proximity space. Let $\varphi: X_{1} \rightarrow X_{2}, \psi: E_{1} \rightarrow E_{2}$ and $\eta: K_{1} \rightarrow K_{2}$ be functions. Then the mapping $\varphi_{\psi, \eta}$ from $\left(X_{1}, \delta^{1}\right)$ into $\left(X_{2}, \delta^{2}\right)$ is called a soft proximally continuous if

$$
\begin{array}{r}
\delta_{k}^{1}(f, g) \leq \delta_{\eta(k)}^{2}\left(\varphi_{\psi}(f), \varphi_{\psi}(g)\right), \quad \text { for each } k \in K_{1}, \\
f, g \in\left(I^{X_{1}}\right)^{E_{1}} .
\end{array}
$$

Lemma 14. Let $\left(X_{1}, \delta^{1}\right)$ and $\left(X_{2}, \delta^{2}\right)$ be an $\left(E_{1}, K_{1}\right)$-soft fuzzy proximity space and an $\left(E_{2}, K_{2}\right)$-soft fuzzy proximity space, respectively. Let $\varphi_{\psi, \eta}$ be a mapping from $\left(X_{1}, \delta^{1}\right)$ into $\left(X_{2}, \delta^{2}\right)$. Then, $\varphi_{\psi, \eta}$ is soft proximally continuous if and only if

$$
\begin{gathered}
\delta_{k}^{1}\left(\varphi_{\psi}^{-1}(u), \varphi_{\psi}^{-1}(v)\right) \leq \delta_{\eta(k)}^{2}(u, v), \\
\text { for each } k \in K_{1}, u, v \in\left(I^{X_{2}}\right)^{E_{2}} .
\end{gathered}
$$


The category of soft fuzzy proximity spaces and soft proximally continuous mappings will be denoted by SFP.

Definition 15. A mapping $\Delta: K \rightarrow 2^{\left(I^{X}\right)^{E} \times\left(I^{X}\right)^{E}}$ is called a Katsaras $(E, K)$-soft fuzzy proximity on a set $X$ if for any $f, g, h \in\left(I^{X}\right)^{E}$ and $k \in K$, the next conditions are satisfied:

(K1) $f \Delta_{k} g$ if and only if $g \Delta_{k} f$;

(K2) $(f \sqcup g) \Delta_{k} h$ if and only if $f \Delta_{k} h$ or $g \Delta_{k} h$;

(K3) if $f \Delta_{k} g$, then $f \neq 0_{X}$ and $g \neq 0_{X}$;

(K4) if $f \bar{\Delta}_{k} g$, then $f \sqsubseteq g^{\prime}$;

(K5) if $f \bar{\Delta}_{k} g$, then there exists $d \in\left(I^{X}\right)^{E}$ such that $f \bar{\Delta}_{k} d$ and $g \bar{\Delta}_{k} d^{\prime}$.

(Here, $\bar{\Delta}_{k}$ denotes the negation of $\Delta_{k}$; we write $f \Delta_{k} g$ as the synonym of $\left.(f, g) \in \Delta_{k}\right)$. A pair $(X, \Delta)$ is called a Katsaras $(E, K)$-soft fuzzy proximity space, where for each $k \in K, \Delta_{k} \subset$ $\left(I^{X}\right)^{E} \times\left(I^{X}\right)^{E}$ is a relation on $\left(I^{X}\right)^{E}$.

A mapping $\varphi_{\psi, \eta}:\left(X_{1}, \Delta^{X_{1}}\right) \rightarrow\left(X_{2}, \Delta^{X_{2}}\right)$ where $\left(X_{1}, \Delta^{X_{1}}\right)$, $\left(X_{2}, \Delta^{X_{2}}\right)$ are Katsaras $\left(E_{i}, K_{i}\right)$-soft fuzzy proximity spaces $(i \in\{1,2\})$, respectively, is called soft proximally continuous if $f \Delta_{k}^{X_{1}} g$ implies $\varphi_{\psi}(f) \Delta_{\eta(k)}^{X_{2}} \varphi_{\psi}(g)$.

The category of Katsaras soft fuzzy proximity spaces and soft proximally continuous mappings will be denoted by KSFP.

It is obvious that KSFP can be identified as the full subcategory of SFP and additionally, the objects of this category satisfy the following axiom:

$$
(K) \delta_{k}\left(\left(I^{X}\right)^{E} \times\left(I^{X}\right)^{E}\right) \subset 2:=\{0,1\}, \quad \text { for each } k \in K \text {. }
$$

Now, we give the generalization of pseudo-fuzzy proximity which is considered by Katsaras as a more restricted concept of a fuzzy proximity in his former papers $[13,14]$ to the soft case. (We use the expression "pseudo-fuzzy" because the "fuzziness" of this relation is rather a poor one: Katsaras pseudo-fuzzy proximities are in a canonical one-to-one correspondence with usual proximities [15]).

Definition 16. A mapping $\Delta: K \rightarrow 2^{\left(I^{X}\right)^{E} \times\left(I^{X}\right)^{E}}$ satisfying (K1), (K2), (K3), (K5), and the following strengthened version of axiom (K4) is called a Katsaras $(E, K)$-soft pseudo-fuzzy proximity on $X$ : if for all $k \in K$,

$$
\left(K 4^{\prime}\right) f \bar{\Delta}_{k} g, \text { then } f \sqcap g=0_{X} .
$$

The corresponding full subcategory of KSFP will be denoted by $\operatorname{KSFP}\left(4^{\prime}\right)$. Obviously $\operatorname{KSFP}\left(4^{\prime}\right)$ can be described as "intersection" of KSFP and SFP.

When studying fuzzy topologies (in the sense of $(0,1))$ it is often useful to apply the technique of representing a general fuzzy topology $\mathscr{T}$ by means of a "continuous" decreasing system $\left\{\mathscr{T}_{\alpha}: \alpha \in(0,1)\right\}$ of its level Chang fuzzy topologies (see [26]). Our next aim is to obtain a similar result for soft fuzzy proximities. For this purpose we will use a slightly modified version of a Katsaras soft fuzzy proximity.
Definition 17. A mapping $\Delta^{\alpha}: K \rightarrow 2^{\left(I^{X}\right)^{E} \times\left(I^{X}\right)^{E}}$ where $\alpha \in I$ is called an $\alpha$-Katsaras $(E, K)$-soft fuzzy proximity on $X$ if it satisfies (K1), (K2), (K3), (K5), and the following axiom: for all $k \in K$, (where $\alpha_{X}(e)=\bar{\alpha}$ for each $\left.e \in E\right)$

$$
\left(K 4_{\alpha}\right) f \bar{\Delta}_{k}^{\alpha} g \text { implies } f \sqcap g \sqsubset \alpha_{X} \text {. }
$$

Proposition 18. Let $(X, \delta)$ be an $(E, K)$-soft fuzzy proximity space and $\alpha \in I$. For $k \in K$ and $f, g \in\left(I^{X}\right)^{E}$ let $f \Delta_{k}^{\alpha} g$ if and only if $\delta_{k}(f, g) \geq \alpha$. Then $\Delta^{\alpha}$ is an $\alpha$-Katsaras $(E, K)$-soft fuzzy proximity on $X$.

Proof. The validity of axioms (K1), (K2), (K3), and (K5) for $\Delta_{k}^{\alpha}$ is obvious. If $f \bar{\Delta}_{k}^{\alpha} g$, then $\sup _{x \in X} \sup _{e \in E}(f \sqcap g)_{e}(x) \leq \delta_{k}(f, g)$ and hence $\left(K 4_{\alpha}\right)$ holds for $\Delta_{k}^{\alpha}$, too.

Let $(X, \delta)$ be an $(E, K)$-soft fuzzy proximity space. For $k \in K$ consider the system $D^{k}=\left\{\Delta_{k}^{\alpha}: \alpha \in(0,1]\right\}$ of $\alpha$ Katsaras soft fuzzy proximities $\Delta_{k}^{\alpha}=\left\{(f, g) \in\left(I^{X}\right)^{E} \times\left(I^{X}\right)^{E}\right.$ : $\left.\delta_{k}(f, g) \geq \alpha\right\}$ on the set $X$. It is easy to notice that this system is nonincreasing; that is, $\alpha^{\prime}<\alpha$ implies $\Delta_{k}^{\alpha^{\prime}} \supset \Delta_{k}^{\alpha}$, and it is lower semicontinuous in the following sense: for each $\alpha \in(0,1]$ it holds $\Delta_{k}^{\alpha}=\bigcap_{\alpha<\alpha^{\prime}} \Delta_{k}^{\alpha^{\prime}}$.

Conversely, for each $k \in K$ let a lower semicontinuous nonincreasing system $D^{k}=\left\{\Delta_{k}^{\alpha}: \alpha \in(0,1]\right\}$ of $\Delta^{\alpha} \alpha$ Katsaras soft fuzzy proximities on a set $X$ be given. For each $k \in K$, define a mapping $\delta_{k}:\left(I^{X}\right)^{E} \times\left(I^{X}\right)^{E} \rightarrow I$ by the equality $\delta_{k}(f, g)=\sup \left\{\Delta_{k}^{\alpha} \wedge \alpha: \alpha \in(0,1]\right\}$. Indeed, axioms (P1), (P2), and (P3) are obvious. To show axiom (P4) assume that $\delta_{k}(f, g)=\alpha$; then $(f, g) \notin \Delta_{k}^{\alpha+\varepsilon}$ for each $\varepsilon>0$ and hence by $\left(K 4_{\alpha}\right)(f \sqcap g)_{e}(x)<\alpha+\varepsilon$, for all $e \in E$. Since $\varepsilon>0$ is arbitrary it follows that $\sup _{x \in X} \sup _{e \in E}(f \sqcap g)_{e}(x) \leq \delta_{k}(f, g)$. To show axiom (P5) find $d_{\varepsilon} \in\left(I^{X}\right)^{E}$ such that $f \bar{\Delta}_{k}^{\alpha+\varepsilon} d_{\varepsilon}$ and $g \bar{\Delta}_{k}^{\alpha+\varepsilon} d_{\varepsilon}^{\prime}$. Then $\delta_{k}\left(f, d_{\varepsilon}\right), \delta_{k}\left(g, d_{\varepsilon}^{\prime}\right)<\alpha+\varepsilon$ and hence $\delta_{k}(f, g)>\inf \left\{\delta_{k}\left(f, d_{\varepsilon}\right) \vee \delta_{k}\left(g, d_{\varepsilon}^{\prime}\right): d_{\varepsilon} \in\left(I^{X}\right)^{E}\right\}-\varepsilon$. Since $\varepsilon>0$ is arbitrary, the validity of axiom (P5) follows from here.

Notice also that the $(E, K)$-soft fuzzy proximity $\delta$ has exactly $\Delta^{\alpha}$ as its $\alpha$-Katsaras $(E, K)$-soft fuzzy proximity: for each $k \in K, \Delta_{k}^{\alpha}=\left\{(f, g): \delta_{k}(f, g) \geq \alpha\right\}$. Indeed the inclusion $\left\{(f, g): \delta_{k}(f, g) \geq \alpha\right\} \supset \Delta_{k}^{\alpha}$ is obvious. To verify the inverse inclusion assume that $\delta_{k}(f, g) \geq \alpha$. Then $(f, g) \in \Delta_{k}^{\alpha^{\prime}}$ for all $\alpha^{\prime}<\alpha$, and, hence, by lower semicontinuity of $D^{k}, f, g \in \Delta_{k}^{\alpha}$, too.

The obtained results can be gathered in the following statement.

Theorem 19. Let $(X, \delta)$ be an $(E, K)$-soft fuzzy proximity space and for each $k \in K$, let $\Delta_{k}^{\alpha}=\left\{(f, g) \in\left(I^{X}\right)^{E} \times\right.$ $\left.\left(I^{X}\right)^{E}: \delta_{k}(f, g) \geq \alpha\right\}$. Then $D^{k}=\left\{\Delta_{k}^{\alpha}: \alpha \in(0,1]\right\}$ is a nonincreasing lower semicontinuous system of $\Delta^{\alpha} \alpha$-Katsaras soft fuzzy proximities. Conversely, for each $k \in K$, given a family of nonincreasing systems $D^{k}=\left\{\Delta_{k}^{\alpha}: \alpha \in(0,1]\right\}$ of $\alpha$-Katsaras soft fuzzy proximities, by the formula $\delta_{k}(f, g)=$ $\sup \left\{\Delta_{k}^{\alpha}(f, g) \wedge \alpha: \alpha \in(0,1]\right\}$, we can define an $(E, K)$-soft fuzzy proximity $\delta$ on $X$. Besides, if $D^{k}$ is lower semicontinuous, then $\Delta_{k}^{\alpha}=\left\{(f, g): \delta_{k}(f, g) \geq \alpha\right\}$. 
Definition 20. Let $\delta, \delta^{*}$ be $(E, K)$-soft fuzzy proximities on a set $X$. We say that $\delta$ is stronger (or smaller) than $\delta^{*}$ denoted by $\delta \leq \delta^{*}$, if $\delta_{k}(f) \leq \delta_{k}^{*}(f)$ for each $k \in K, f \in\left(I^{X}\right)^{E}$. In this case we also say that $\delta^{*}$ is weaker (or larger) than $\delta$. It is obvious that $\delta_{k} \leq \delta_{k}^{*}$ if and only if $\Delta_{k}^{\alpha} \subset \Delta_{k}^{* \alpha}$ for each $k \in K$, $\alpha \in(0,1]$, where $\Delta^{\alpha}$ and $\Delta^{* \alpha}$ are the corresponding level $\alpha$ Katsaras soft fuzzy proximities.

Different from the situation in crisp mathematics where each category has a strictly determined class of morphisms, in fuzzy mathematics it is sometimes desirable to introduce a "measure of defectiveness" for "potential morphisms." Below we define the defect of soft proximal continuity for a mapping of soft fuzzy proximity spaces.

Definition 21. By the defect of soft proximal continuity of a mapping $\varphi_{\psi, \eta}$ from $\left(X_{1}, \delta^{1}\right)$ to $\left(X_{2}, \delta^{2}\right)$, where $\left(X_{1}, \delta^{1}\right)$ is an $\left(E_{1}, K_{1}\right)$-soft fuzzy proximity space and $\left(X_{2}, \delta^{2}\right)$ is an $\left(E_{2}, K_{2}\right)$-soft fuzzy proximity space, we call the number

$$
\operatorname{pd}\left(\varphi_{\psi, \eta}\right)=\sup _{k \in K_{1}, g \in\left(I^{X}\right)^{E}} \sup _{k}\left(\delta_{k}^{1}(f, g)-\delta_{\eta(k)}^{2}\left(\varphi_{\psi}(f), \varphi_{\psi}(g)\right)\right) .
$$

Obviously, $0 \leq \operatorname{pd}\left(\varphi_{\psi, \eta}\right) \leq 1$ for each mapping $\varphi_{\psi, \eta}$. If $\varphi_{\psi, \eta}$ is soft proximally continuous, then $\operatorname{pd}\left(\varphi_{\psi, \eta}\right)=0$. If $\left(X_{1}, \delta^{1}\right)$ and $\left(X_{2}, \delta^{2}\right)$ are Katsaras soft fuzzy proximity spaces and $\varphi_{\psi, \eta}$ is not proximally continuous, then $\operatorname{pd}\left(\varphi_{\psi, \eta}\right)=1$. However, in case of general soft fuzzy proximity spaces $\operatorname{pd}\left(\varphi_{\psi, \eta}\right)$ may be any number in $[0,1]$.

Proposition 22. If $\varphi_{\psi, \eta}:\left(X_{1}, \delta^{1}\right) \rightarrow\left(X_{2}, \delta^{2}\right)$ and $\varphi_{\psi^{*}, \eta^{*}}^{*}:$ $\left(X_{2}, \delta^{2}\right) \rightarrow\left(X_{3}, \delta^{3}\right)$ are mappings of $\left(E_{i}, K_{i}\right)$-soft fuzzy proximity spaces $\left(X_{i}, \delta^{i}\right), i \in\{1,2,3\}$, respectively, then $\operatorname{pd}\left(\varphi_{\psi^{*}, \eta^{*}}^{*} \circ \varphi_{\psi, \eta}\right) \leq \operatorname{pd}\left(\varphi_{\psi, \eta}\right)+\operatorname{pd}\left(\varphi_{\psi^{*}, \eta^{*}}^{*}\right)$.

Proof. Consider

$$
\begin{aligned}
& \operatorname{pd}\left(\varphi_{\psi^{*}, \eta^{*}}^{*} \circ \varphi_{\psi, \eta}\right) \\
& =\sup _{k \in K_{1}} \sup _{f, g \in\left(I^{X_{1}}\right)^{E_{1}}}\left(\delta_{k}^{1}(f, g)-\delta_{\eta^{*} \circ \eta(k)}^{3}\left(\left(\varphi^{*} \circ \varphi\right)_{\psi^{*} \circ \psi}(f),\right.\right. \\
& \left.\left.\left(\varphi^{*} \circ \varphi\right)_{\psi^{*} \circ \psi}(g)\right)\right) \\
& =\sup _{k \in K_{1}} \sup _{f, g \in\left(I^{X_{1}}\right)^{E^{1}}}\left(\delta_{k}^{1}(f, g)\right. \\
& -\delta_{\eta(k)}^{2}\left(\varphi_{\psi}(f), \varphi_{\psi}(g)\right) \\
& +\delta_{\eta(k)}^{2}\left(\varphi_{\psi}(f), \varphi_{\psi}(g)\right) \\
& -\delta_{\eta^{*} \circ \eta(k)}^{3}\left(\left(\varphi^{*} \circ \varphi\right)_{\psi^{*} \circ \psi}(f),\right. \\
& \left.\left.\left(\varphi^{*} \circ \varphi\right)_{\psi^{*} \circ \psi}(g)\right)\right) \\
& \leq \sup _{k \in K_{1}} \sup _{f, g \in\left(I^{X}\right)^{E}}\left(\delta_{k}^{1}(f, g)-\delta_{\eta(k)}^{2}\left(\varphi_{\psi}(f), \varphi_{\psi}(g)\right)\right) \\
& +\sup _{k \in K_{1} h, d \in\left(I^{X_{2}}\right)^{E_{2}}} \sup _{\eta(k)}\left(\delta^{2}(h, d)-\delta_{\eta^{*}(\eta(k))}^{3}\left(\varphi_{\psi^{*}}^{*}(h), \varphi_{\psi^{*}}^{*}(d)\right)\right)
\end{aligned}
$$

$$
\begin{aligned}
\leq & \sup _{k \in K_{1}} \sup _{f, g \in\left(I^{X}\right)^{E}}\left(\delta_{k}^{1}(f, g)-\delta_{\eta(k)}^{2}\left(\varphi_{\psi}(f), \varphi_{\psi}(g)\right)\right) \\
& +\sup _{l \in K_{2} h, d \in\left(I^{X_{2}}\right)^{E_{2}}} \sup _{l}\left(\delta_{l}^{2}(h, d)-\delta_{\eta^{*}(l)}^{3}\left(\varphi_{\psi^{*}}^{*}(h), \varphi_{\psi^{*}}^{*}(d)\right)\right) \\
= & \operatorname{pd}\left(\varphi_{\psi, \eta}\right)+\operatorname{pd}\left(\varphi_{\psi^{*}, \eta^{*}}^{*}\right) .
\end{aligned}
$$

\section{Soft Fuzzy Topologies Generated by Soft Fuzzy Proximity}

In this section, we generate a soft fuzzy topology from a given soft fuzzy proximity by using the closure operator.

Let $(X, \delta)$ be an $(E, K)$-soft fuzzy proximity space, $f \in$ $\left(I^{X}\right)^{E}$, and $\alpha \in(0,1]$. The $\alpha$-closure of the fuzzy soft set $f$ (or the closure of $f$ at the level $\alpha$ ) with respect to the parameter $k \in K$ is defined by the equality $\mathscr{C}(k, f, \alpha)=$ $\left(\bigsqcup\left\{g: \delta_{k}(f, g) \leq \alpha^{\prime}\right\}\right)^{\prime} \sqcup f=\left(\prod\left\{g^{\prime}: \delta_{k}(f, g) \leq \alpha^{\prime}\right\}\right) \sqcup f$.

Lemma 23. Consider $\mathscr{C}(k, f, \alpha)=\left(\bigsqcup\left\{P: \delta_{k}\left(P^{*}, f\right)>\alpha^{\prime}\right\}\right) \sqcup$ $f\left(\right.$ where $P(e)=x^{\lambda}$ and $\left.P^{*}(e)=x^{\lambda^{\prime}}\right)$.

Proof. To show $\mathscr{C}(k, f, \alpha) \sqsubseteq\left(\bigsqcup\left\{P: \delta_{k}\left(P^{*}, f\right)>\alpha^{\prime}\right\}\right) \sqcup f$, take $e \in E$ and $x^{\mu} \notin \bigvee\left\{x^{\lambda}: \delta_{k}\left(P^{*}, f\right)>\alpha^{\prime}\right\}$ and choose $\varepsilon>0$ such that $Q(e)=x^{\mu-\varepsilon} \notin \bigvee\left\{x^{\lambda}: \delta_{k}\left(P^{*}, f\right)>\alpha^{\prime}\right\}$. Then $\delta_{k}\left(Q^{*}, f\right) \leq \alpha^{\prime}$, and hence $\left(x^{\mu^{\prime}+\varepsilon}\right)^{\prime} \geq \bigwedge\left\{g^{\prime}(e): \delta_{k}(f, g) \leq\right.$ $\left.\alpha^{\prime}\right\}$. Since obviously $x^{\mu} \notin\left(x^{\mu^{\prime}+\varepsilon}\right)^{\prime}$, we conclude that $x^{\mu} \notin$ $\bigwedge\left\{g^{\prime}(e): \delta_{k}(f, g) \leq \alpha^{\prime}\right\}$.

To show the inverse inequality take $e \in E$ and $Q(e)=$ $x^{\mu} \in\left(\bigvee\left\{x^{\lambda}: \delta_{k}\left(P^{*}, f\right)>\alpha^{\prime}\right\}\right) \vee f_{e}$. Besides, without loss of generality we may assume that $\left(\left(\bigsqcup\left\{P: \delta_{k}\left(P^{*}, f\right)>\right.\right.\right.$ $\left.\left.\left.\alpha^{\prime}\right\}\right) \sqcup f\right)_{e}(x)>\mu$, for all $e \in E, x \in X$ (and not only $\left(\left(\bigsqcup\left\{P: \delta_{k}\left(P^{*}, f\right)>\alpha^{\prime}\right\}\right) \sqcup f\right)_{e}(x) \geq \mu$, for all e $\in E, x \in X$ (and not only $\left.\left(\left(\bigsqcup\left\{P: \delta_{k}\left(P^{*}, f\right)>\alpha^{\prime}\right\}\right) \sqcup f\right)_{e}(x) \geq \mu\right)$ and that $x^{\mu} \notin f$. However this implies that $\delta_{k}\left(Q^{*}, f\right)>\alpha^{\prime}$. Hence, if $\delta_{k}(g, f) \leq \alpha^{\prime}$ for some $g \in\left(I^{X}\right)^{E}$, then $g_{e}^{\prime}(x)>\mu$. Thus, $x^{\mu} \in g^{\prime}(e)$ for each $g$ satisfying the inequality $\delta_{k}(f, g) \leq \alpha^{\prime}$ and hence $x^{\mu} \in \bigwedge\left\{g^{\prime}(e): \delta_{k}(f, g) \leq \alpha^{\prime}\right\}$.

Proposition 24. Let $(X, \delta)$ be an $(E, K)$-soft fuzzy proximity space. Define the mapping $\mathscr{C}: K \times\left(I^{X}\right)^{E} \times(0,1] \rightarrow\left(I^{X}\right)^{E}$ by

$$
\begin{aligned}
\mathscr{C}(k, f, \alpha) & =\left(\bigsqcup\left\{g: \delta_{k}(f, g) \leq \alpha^{\prime}\right\}\right)^{\prime} \sqcup f \\
& =\left(\prod\left\{g^{\prime}: \delta_{k}(f, g) \leq \alpha^{\prime}\right\}\right) \sqcup f .
\end{aligned}
$$

Then the mapping $\mathscr{C}$ is a topological $(E, K)$-soft fuzzy closure operator.

Proof. (C1) $\mathscr{C}\left(k, 0_{X}, \alpha\right)=\left(\prod\left\{g^{\prime}: \delta_{k}\left(0_{X}, g\right) \leq \alpha^{\prime}\right\}\right) \sqcup 0_{X}=$ $0_{X} \sqcup 0_{X}=0_{X}$.

(C2) It is obvious that $f \sqsubseteq \mathscr{C}(k, f, \alpha)$. 
(C3) Let $f, h \in\left(I^{X}\right)^{E}$ and $f \sqsubseteq h$. Since $\mathscr{C}(k, f, \alpha)=$ $\left(\prod\left\{g^{\prime}: \delta_{k}(f, g) \leq \alpha^{\prime}\right\}\right) \sqcup f, \mathscr{C}(k, h, \alpha)=\left(\prod\left\{g^{\prime}: \delta_{k}(h, g) \leq\right.\right.$ $\left.\left.\alpha^{\prime}\right\}\right) \sqcup h$ and $\delta_{k}(f, g) \leq \delta_{k}(h, g)$, then we have $\mathscr{C}(k, f, \alpha)$ $\mathscr{C}(k, h, \alpha)$.

(C4) For $\alpha \leq \beta$, since $\delta_{k}(f, g) \leq \beta^{\prime} \leq \alpha^{\prime}$, we have $\mathscr{C}(k, f, \alpha) \sqsubseteq \mathscr{C}(k, f, \beta)$.

(C5) Applying axiom (P3), we get $\mathscr{C}(k, f \sqcup g, \alpha)=$ $\left(\bigsqcup\left\{h: \delta_{k}(f \sqcup g, h) \leq \alpha^{\prime}\right\}\right)^{\prime} \sqcup(f \sqcup g)=\left(\bigsqcup\left\{h: \delta_{k}(f, h) \vee\right.\right.$ $\left.\left.\delta_{k}(g, h) \leq \alpha^{\prime}\right\}\right)^{\prime} \sqcup(f \sqcup g)=\left(\left(\bigsqcup\left\{h_{1}: \delta_{k}\left(f, h_{1}\right) \leq \alpha^{\prime}\right\}\right) \sqcap\left(\bigsqcup\left\{h_{2}:\right.\right.\right.$ $\left.\left.\left.\delta_{k}\left(g, h_{2}\right) \leq \alpha^{\prime}\right\}\right)\right)^{\prime} \sqcup(f \sqcup g)=\left(\left(\bigsqcup\left\{h_{1}: \delta_{k}\left(f, h_{1}\right) \leq \alpha^{\prime}\right\}\right)^{\prime} \sqcup f\right) \sqcup$ $\left(\left(\bigsqcup\left\{h_{2}: \delta_{k}\left(g, h_{2}\right) \leq \alpha^{\prime}\right\}\right)^{\prime} \sqcup g\right)=\mathscr{C}(k, f, \alpha) \sqcup \mathscr{C}(k, g, \alpha)$.

Hence, $\mathscr{C}$ is an $(E, K)$-soft fuzzy closure operator.

Let $k \in K, f, g \in\left(I^{X}\right)^{E}$ and let $\delta_{k}(f, g) \leq \alpha^{\prime}$ be given. By (P5), $\inf _{h \in\left(I^{X}\right)^{E}}\left\{\delta_{k}(f, h) \vee \delta_{k}\left(h^{\prime}, g\right)\right\} \leq \delta_{k}(f, g) \leq \alpha^{\prime}$. Then for each $\varepsilon>0$, there exists $h_{\varepsilon} \in\left(I^{X}\right)^{E}$ such that $\delta_{k}\left(f, h_{\varepsilon}\right) \vee$ $\delta_{k}\left(h_{\varepsilon}^{\prime}, g\right)<\alpha^{\prime}+\varepsilon$. Hence $\delta_{k}\left(f, h_{\varepsilon}\right)<\alpha^{\prime}+\varepsilon$ and $\delta_{k}\left(h_{\varepsilon}^{\prime}, g\right)<\alpha^{\prime}+$ $\varepsilon$. This implies $\mathscr{C}(k, f, \alpha-\varepsilon) \sqsubseteq h_{\varepsilon}^{\prime}$. Hence $\delta_{k}(g, \mathscr{C}(k, f, \alpha-\varepsilon)) \leq$ $\delta_{k}\left(h_{\varepsilon}^{\prime}, g\right)<\alpha^{\prime}+\varepsilon$. Here $\delta_{k}(f, g) \leq \alpha^{\prime}$ implies $\delta_{k}(g, \mathscr{C}(k, f, \alpha-$ $\varepsilon))<\alpha^{\prime}+\varepsilon$, for all $\varepsilon>0$. Then we have

$$
\begin{aligned}
\mathscr{C}(k, f, \alpha) & =\left(\prod\left\{g^{\prime}: \delta_{k}(f, g) \leq \alpha^{\prime}\right\}\right) \sqcup f \\
& =\left(\prod\left\{g^{\prime}: \delta_{k}(f, g) \leq \alpha^{\prime}\right\}\right) \sqcup \mathscr{C}(k, f, \alpha) \\
& \sqsupseteq\left(\prod\left\{g^{\prime}: \delta_{k}(\mathscr{C}(k, f, \alpha-\varepsilon), g)<\alpha^{\prime}+\varepsilon\right\}\right) \\
& \sqcup \mathscr{C}(k, f, \alpha) .
\end{aligned}
$$

If we take limit for $\varepsilon \rightarrow 0$ in the last step of the inequality, then we have $\mathscr{C}(k, f, \alpha) \sqsupseteq \mathscr{C}(k, \mathscr{C}(k, f, \alpha), \alpha)$. Hence the proof is complete.

Notation. Let $\sigma_{\alpha}^{k}=\left\{f \in\left(I^{X}\right)^{E}: f=\mathscr{C}(k, f, \alpha)\right\}$ and $\mathscr{T}_{\alpha}^{k}=$ $\left\{f: f^{\prime} \in \sigma_{\alpha}^{k}\right\}$. It is easy to verify that for each $k \in K, \mathscr{T}_{\alpha}^{k}$ is a topology of fuzzy soft sets in the sense of Tanay and Kandemir [27].

Proposition 25. The (E, K)-soft fuzzy closure operator $\mathscr{C}$ is continuous along I in the following sense:

$$
\begin{aligned}
& \text { if } \varepsilon_{n} \longrightarrow 0, \varepsilon_{n}>0, \\
& \text { then } \bigsqcup_{n} \mathscr{C}\left(k, f, \alpha-\varepsilon_{n}\right)=\mathscr{C}(k, f, \alpha), \quad \forall k \in K .
\end{aligned}
$$

Proof. Since for each $k \in K, \mathscr{C}\left(k, f, \alpha-\varepsilon_{n}\right) \sqsubseteq \mathscr{C}(k, f, \alpha)$.

It follows that $\bigsqcup_{n} \mathscr{C}\left(k, f, \alpha-\varepsilon_{n}\right) \sqsubseteq \mathscr{C}(k, f, \alpha)$, for all $k \in K$. To prove the converse inequality it is sufficient to show that $\prod_{n}\left(\bigsqcup\left\{g_{n}: \delta_{k}\left(f, g_{n}\right) \leq \alpha^{\prime}+\varepsilon_{n}\right\}\right) \sqsubseteq \bigsqcup\left\{g: \delta_{k}(f, g) \leq \alpha^{\prime}\right\}$. Assume, contrary, that there exist $x \in X, e \in E$ and $\gamma>0$ such that $\prod_{n}\left(\bigsqcup\left\{g_{n}: \delta_{k}\left(f, g_{n}\right) \leq \alpha^{\prime}+\varepsilon_{n}\right\}\right)_{e}(x)>\left(\bigsqcup\left\{g: \delta_{k}(f, g) \leq\right.\right.$ $\left.\left.\alpha^{\prime}\right\}\right)_{e}(x)+\gamma$. Then for each $n \in \mathbb{N}$ one can find $g_{n}$ such that $\delta_{k}\left(f, g_{n}\right) \leq \alpha^{\prime}+\varepsilon_{n}$ and $\left(g_{n}\right)_{e}(x)>\left(\bigsqcup\left\{g: \delta_{k}(f, g) \leq\right.\right.$ $\left.\left.\alpha^{\prime}\right\}\right)_{e}(x)+\gamma$. Now, denoting $g^{0}=\prod_{n} g_{n}$ we have $\delta_{k}\left(f, g^{0}\right) \leq \alpha^{\prime}$ and $g_{e}^{0}(x) \geq\left(\bigsqcup\left\{g: \delta_{k}(f, g) \leq \alpha^{\prime}\right\}\right)_{e}(x)+\gamma$. The obtained contradiction completes the proof.
Proposition 26. For each $\alpha \in(0,1]$ and $k \in K, \sigma_{\alpha}^{k}=$ $\bigcap_{\alpha^{\prime}<\alpha} \sigma_{\alpha^{\prime}}^{k}$ and hence $\mathscr{T}_{\alpha}^{k}=\bigcap_{\alpha^{\prime}<\alpha} \mathscr{T}_{\alpha^{\prime}}^{k}$

Proof. It is obvious that $\alpha^{\prime}<\alpha$ implies $\mathscr{C}\left(k, f, \alpha^{\prime}\right) \sqsubseteq \mathscr{C}(k$, $f, \alpha)$ for each $f \in\left(I^{X}\right)^{E}$ and hence $\sigma_{\alpha}^{k} \subseteq \sigma_{\alpha^{\prime}}^{k}$. Take now $f \in$ $\bigcap_{\alpha^{\prime}<\alpha} \sigma_{\alpha^{\prime}}^{k}$ and consider a decreasing sequence $\varepsilon_{n} \rightarrow 0$. Then $f=\mathscr{C}\left(k, f, \alpha-\varepsilon_{n}\right)$ for each $n \in \mathbb{N}$. Applying Proposition 25, we get $\mathscr{C}(k, f, \alpha)=\bigsqcup_{n} \mathscr{C}\left(k, f, \alpha-\varepsilon_{n}\right)=f$; that is, $f \in \sigma_{\alpha}^{k}$.

From this proposition, similarly as in Theorem 2.6 [17], we have the following.

Theorem 27. The mapping $\tau: K \rightarrow I^{\left(I^{X}\right)^{E}}$ defined by the equality, for each $k \in K, \tau_{k}(f)=\bigvee_{\alpha}\left(\mathscr{T}_{\alpha}^{k}(f) \wedge \alpha\right)$ is an $(E, K)$ soft fuzzy topology on X. Besides $\mathscr{T}_{\alpha}^{k}=\left\{f: \tau_{k}(f) \geq \alpha\right\}$.

The (E, K)-soft fuzzy topology constructed in Theorem 27 will be called generated by $\delta$ and, if necessary, will be denoted by $\tau_{\delta}$.

Theorem 28. If a mapping $\varphi_{\psi, \eta}:\left(X_{1}, \delta^{1}\right) \rightarrow\left(X_{2}, \delta^{2}\right)$ is soft proximally continuous, then the mapping $\varphi_{\psi, \eta}:\left(X_{1}, \tau_{\delta^{1}}\right) \rightarrow$ $\left(X_{2}, \tau_{\delta^{2}}\right)$ is soft fuzzy continuous.

Proof. Similarly to the proof of Theorem 2.7 in [17], it is sufficient to verify the inequality $\varphi_{\psi}(\mathscr{C}(k, f, \alpha)) \sqsubseteq \mathscr{C}(\eta(k)$, $\left.\varphi_{\psi}(f), \alpha\right)$ for each $\alpha \in(0,1]$. From Lemma 23 it follows that $\varphi_{\psi}(\mathscr{C}(k, f, \alpha))_{e_{2}}=\varphi_{\psi}\left(\bigsqcup\left\{P: \delta_{k}^{1}\left(P^{*}, f\right)>\alpha^{\prime}\right\} \sqcup f\right)_{e_{2}}=$ $\left(\bigsqcup\left\{\varphi_{\psi}\left(x^{\lambda}\right): \delta_{k}^{1}\left(P^{*}, f\right)>\alpha^{\prime}\right\}\right) \sqcup \varphi_{\psi}(f)_{e_{2}}=\left(\bigsqcup\left\{(\varphi(x))^{\lambda}:\right.\right.$ $\left.\left.\delta_{k}^{1}\left(P^{*}, f\right)>\alpha^{\prime}\right\}\right) \sqcup \varphi_{\psi}(f)$. Since $\varphi_{\psi, \eta}$ is soft proximally continuous it is $\delta_{k}^{1}\left(P^{*}, f\right) \leq \delta_{\eta(k)}^{2}\left(\varphi_{\psi}(f), \varphi_{\psi}(P)^{*}\right)$ and hence $\varphi_{\psi}(\mathscr{C}(k, f, \alpha))_{e_{2}} \leq \bigvee\left\{(\varphi(x))^{\lambda}: \delta_{\eta(k)}^{2}\left(\varphi_{\psi}(f),(\varphi(P))^{*}\right)>\alpha^{\prime}\right\} \sqcup$ $\varphi_{\psi}(f)_{e_{2}} \leq \bigvee\left\{Q\left(e_{2}\right): \delta_{\eta(k)}^{2}\left(\varphi_{\psi}(f), Q^{*}\right)>\alpha^{\prime}\right\} \sqcup \varphi_{\psi}(f)_{e_{2}}=$ $\mathscr{C}\left(\eta(k), \varphi_{\psi}(f), \alpha\right)_{e_{2}}$.

From Theorems 27 and 28 it follows that the procedure assigning to each soft fuzzy proximity $\delta$ generated soft fuzzy topology $\tau_{\delta}$ and leaving morphisms unchanged can be interpreted as a functor.

Corollary 29. By letting $\Phi(X, \delta)=\left(X, \tau_{\delta}\right)$ for every soft fuzzy proximity space $(X, \delta)$ and $\Phi\left(\varphi_{\psi, \eta}\right)=\varphi_{\psi, \eta}:\left(X_{1}, \tau_{\delta^{1}}\right) \rightarrow$ $\left(X_{2}, \tau_{\delta^{2}}\right)$ for every soft proximally continuous mappings $\varphi_{\psi, \eta}$ : $\left(X_{1}, \delta^{1}\right) \rightarrow\left(X_{2}, \delta^{2}\right)$, a functor $\Phi$ from the category SFP into SFTOP of soft fuzzy topological spaces.

Proposition 30. If $\delta, \delta^{*}$ are $(E, K)$-soft fuzzy proximities on $X$ and $\delta \leq \delta^{*}$, then $\tau_{\delta} \geq \tau_{\delta^{*}}$.

Proof. It is easy and therefore omitted.

\section{Initial Soft Fuzzy Proximity}

The main aim of this section is to describe products in the category SFP. This description is based on the construction of initial soft fuzzy topologies given below, which has an interest of its own.

Definition 31. Let $X$ be a set, let $E, K$ be the parameter sets, let $\left\{\left(X_{\gamma}, \delta^{\gamma}\right)\right\}_{\gamma \in \Gamma}$ be a family of $\left(E_{\gamma}, K_{\gamma}\right)$-soft fuzzy proximity 
spaces, and let $\left(\varphi_{\psi, \eta}\right)_{\gamma}: X \rightarrow\left(X_{\gamma}, \delta^{\gamma}\right), \gamma \in \Gamma$ be a family of mappings. The weakest soft fuzzy proximity $\delta$ on $X$ for which all mappings $\left(\varphi_{\psi, \eta}\right)_{\gamma}:(X, \delta) \rightarrow\left(X_{\gamma}, \delta^{\gamma}\right), \gamma \in \Gamma$, are soft proximally continuous, is called the initial soft fuzzy proximity for this family of mappings.

The existence of the initial soft fuzzy proximity is provided by the next theorem, containing also its effective characterizations.

Theorem 32. Let $X$ be a set, let $E, K$ be the parameter sets, let $\left\{\left(X_{\gamma}, \delta^{\gamma}\right)\right\}_{\gamma \in \Gamma}$ be a family of $\left(E_{\gamma}, K_{\gamma}\right)$-soft fuzzy proximity spaces, and let $\left(\varphi_{\psi, \eta}\right)_{\gamma}: X \rightarrow\left(X_{\gamma}, \delta^{\gamma}\right), \gamma \in \Gamma$ be a family of mappings. Then the equality

$$
\begin{gathered}
\delta_{k}(f, g) \\
=\bigwedge\left\{\bigvee_{i, j} \bigwedge_{\gamma} \delta_{\eta_{\gamma}(k)}^{\gamma}\left(\left(\varphi_{\psi}\right)_{\gamma}\left(f_{i}\right),\left(\varphi_{\psi}\right)_{\gamma}\left(g_{j}\right)\right) \mid\right. \\
\left.f=\bigsqcup_{i=1}^{n} f_{i}, g=\bigsqcup_{i=1}^{n} g_{i}, n \in \mathbb{N}\right\},
\end{gathered}
$$

where $f, g \in\left(I^{X}\right)^{E}$ and $k \in K$ defines the initial for this family of mappings (E, K)-soft fuzzy proximity $\delta: K \rightarrow I^{\left(I^{X}\right)^{E} \times\left(I^{X}\right)^{E}}$.

Proof. Notice first that for each $k \in K, \delta_{k}(f, g) \geq \beta$ (where $\beta \in I$ ) if and only if for any finite covers $f=\bigsqcup_{i=1}^{n} f_{i}$, $g=\bigsqcup_{j=1}^{l} g_{j}\left(f_{i}, g_{j} \in\left(I^{X}\right)^{E}\right)$ of $f$ and $g$, respectively, there exist $i_{0} \in\{1, \ldots, n\}$ and $j_{0} \in\{1, \ldots, l\}$ such that $\delta_{\eta_{\gamma}(k)}^{\gamma}\left(\left(\varphi_{\psi}\right)_{\gamma}\left(f_{i_{0}}\right),\left(\varphi_{\psi}\right)_{\gamma}\left(g_{j_{0}}\right)\right) \geq \beta$ for all $\gamma \in \Gamma$. Besides, without loss of generality we can assume that $n=l$.

Passing to the proof we shall first establish that $\delta$ thus defined is indeed an $(E, K)$-soft fuzzy proximity. It is obvious that for each $k \in K, \delta_{k}\left(0_{X}, 1_{X}\right)=0$ and $\delta_{k}(f, g)=\delta_{k}(g, f)$. To show that $\delta$ satisfies the third axiom notice first that $g \sqcup h \sqsupseteq g$ and $g \sqcup h \sqsupseteq h$ imply the inequality $\delta_{k}(f, g \sqcup h) \geq \delta_{k}(f, g) \vee$ $\delta_{k}(f, h)$. Assume that $\delta_{k}(f, g \sqcup h)>\beta>\delta_{k}(f, g) \vee \delta_{k}(f, h)$ and find covers $f=\bigsqcup_{i=1}^{n} f_{i}, f=\bigsqcup_{i=1}^{l} f_{i}^{*}, g=\bigsqcup_{i=1}^{n} g_{i}$, $h=\bigsqcup_{i=1}^{l} h_{i}$ such that $\sup _{i, j} \inf _{\gamma} \delta_{\eta_{\gamma}(k)}^{\gamma}\left(\left(\varphi_{\psi}\right)_{\gamma}\left(f_{i}\right),\left(\varphi_{\psi}\right)_{\gamma}\left(g_{j}\right)\right) \vee$ $\sup _{i, j} \inf _{\gamma} \delta_{\eta_{\gamma}(k)}^{\gamma}\left(\left(\varphi_{\psi}\right)_{\gamma}\left(f_{i}^{*}\right),\left(\varphi_{\psi}\right)_{\gamma}\left(h_{j}\right)\right)<\beta$.

Besides, without loss of generality, we may assume that $f_{i}=f_{i}^{*}$ (otherwise take the cover $f=\bigsqcup_{i, j} f_{i j}$, where $f_{i j}=$ $\left.f_{i} \sqcap f_{j}^{*}\right)$.

Over designating the fuzzy soft sets constituting the covers, for each $j \in\{1, \ldots, n\}$ let $g_{n+1}=h_{j}$. Then $g \sqcup h=$ $\bigsqcup_{j=1}^{2 n} g_{j}$ and thus from the above inequality we get

$$
\begin{aligned}
\sup _{i, j \in\{1, \ldots, n\}} & \inf _{\gamma} \delta_{\eta_{\gamma}(k)}^{\gamma}\left(\left(\varphi_{\psi}\right)_{\gamma}\left(f_{i}\right),\left(\varphi_{\psi}\right)_{\gamma}\left(g_{j}\right)\right) \\
& \vee \sup _{i, j \in\{1, \ldots, n\}} \inf _{\gamma} \delta_{\eta_{\gamma}(k)}^{\gamma}\left(\left(\varphi_{\psi}\right)_{\gamma}\left(f_{i}\right),\left(\varphi_{\psi}\right)_{\gamma}\left(h_{j}\right)\right) \\
< & \beta \\
< & \sup _{i \in\{1, . ., n\}, j \in\{1, \ldots, 2 n\}} \inf _{\gamma} \delta_{\eta_{\gamma}(k)}^{\gamma}\left(\left(\varphi_{\psi}\right)_{\gamma}\left(f_{i}\right),\left(\varphi_{\psi}\right)_{\gamma}\left(g_{j}\right)\right) .
\end{aligned}
$$

From the right side of the inequality it follows that there exist $i \in\{1, \ldots, n\}$ and $j \in\{1, \ldots, 2 n\}$ such that $\delta_{\eta_{\gamma}(k)}^{\gamma}\left(\left(\varphi_{\psi}\right)_{\gamma}\left(f_{i}\right),\left(\varphi_{\psi}\right)_{\gamma}\left(g_{j}\right)\right)>\beta$ for all $\gamma \in \Gamma$. Considering the two possible cases, $1 \leq j \leq n$ and $n+1 \leq j \leq 2 n$, we easily obtain contradiction with the left side of the inequality.

To show the fourth axiom assume that $\delta_{k}(f, g)<$ $\sup _{x} \sup _{e \in E}(f \sqcap g)_{e}(x)$ for some $f, g \in\left(I^{X}\right)^{E}$ and find covers $f=\bigsqcup_{i=1}^{n} f_{i}, g=\bigsqcup_{i=1}^{n} g_{i}$ and $\gamma \in \Gamma$ such that $\delta_{\eta_{\gamma}(k)}^{\gamma}\left(\left(\varphi_{\psi}\right)_{\gamma}\left(f_{i}\right),\left(\varphi_{\psi}\right)_{\gamma}\left(g_{j}\right)\right)<\sup _{x} \sup _{e \in E}(f \sqcap g)_{e}(x)$ for all $i, j$. It follows from here that $\delta_{\eta_{\gamma}(k)}^{\gamma}\left(\left(\varphi_{\psi}\right)_{\gamma}(f),\left(\varphi_{\psi}\right)_{\gamma}(g)\right)<$ $\sup _{x} \sup _{e \in E}(f \sqcap g)_{e}(x)$. However, this is impossible, because $\delta^{\gamma}$ is an $\left(E_{\gamma}, K_{\gamma}\right)$-soft fuzzy proximity.

To establish the last axiom fix $\beta>0$ and consider the set $\Sigma_{\beta}^{k}$ of all pairs $(f, g) \in\left(I^{X}\right)^{E} \times\left(I^{X}\right)^{E}$ such that $\delta_{k}(f, g)<\beta$ and $\inf \left\{\delta_{k}(f, h) \vee \delta_{k}\left(g, h^{\prime}\right): h \in\left(I^{X}\right)^{E}\right\} \geq \beta$. The validity of (P5) will follow from the fact that for each $k \in K, \Sigma_{\beta}^{k}$ is empty, in which we are going to establish.

Assume, contrary, that there exists $(f, g) \in \Sigma_{\beta}^{k}$ and notice first that in this case $\delta_{\eta_{\gamma}(k)}^{\gamma}\left(\left(\varphi_{\psi}\right)_{\gamma}(f),\left(\varphi_{\psi}\right)_{\gamma}(g)\right) \geq \beta$ for each $\gamma \in \Gamma$. Indeed, take $d \in\left(I^{X_{\gamma}}\right)^{E_{\gamma}}$ and let $h=$ $\left(\varphi_{\psi}\right)_{\gamma}^{-1}(d)$. If $\delta_{k}(f, h) \geq \beta$, then $\delta_{\eta_{\gamma}(k)}^{\gamma}\left(\left(\varphi_{\psi}\right)_{\gamma}(f),\left(\varphi_{\psi}\right)_{\gamma}(h)\right) \geq \beta$ and hence $\delta_{\eta_{\gamma}(k)}^{\gamma}\left(\left(\varphi_{\psi}\right)_{\gamma}(f), d\right) \geq \beta$. Similarly, if $\delta_{k}\left(g, h^{\prime}\right) \geq$ $\beta$, then $\delta_{\eta_{\gamma}(k)}^{\gamma}\left(\left(\varphi_{\psi}\right)_{\gamma}(g), d^{\prime}\right) \geq \beta$. Recalling the definition of $\Sigma_{\beta}^{k}$ and applying axiom (P5) to $\delta^{\gamma}$ we conclude that $\delta_{\eta_{\gamma}(k)}^{\gamma}\left(\left(\varphi_{\psi}\right)_{\gamma}(f),\left(\varphi_{\psi}\right)_{\gamma}(g)\right) \geq \beta$.

For each $(f, g) \in \Sigma_{\beta}^{k}$ there exist numbers $n, l \in \mathbb{N}$ and covers $f=\bigsqcup_{i=1}^{n} f_{i}$ and $g=\bigsqcup_{j=1}^{l} g_{j}$ such that for each pair $(i, j) \in\{1, \ldots, n\} \times\{1, \ldots, l\}$ one can find $\gamma \in \Gamma$ for which $\delta_{\eta_{\gamma}(k)}^{\gamma}\left(\left(\varphi_{\psi}\right)_{\gamma}\left(f_{i}\right),\left(\varphi_{\psi}\right)_{\gamma}\left(g_{j}\right)\right)<\beta$. Besides, we can assume the fuzzy soft sets $f, g$ and their covers are chosen in such a way that the corresponding sum $n+1$ is the minimal one and that $n \geq 2$. Let $h=f_{1} \sqcup \cdots \sqcup f_{n-1}$. Then one of the following two possibilities should be true:

(a) for every $d \in\left(I^{X}\right)^{E}$ either $\delta_{k}(h, d)>\beta$ or $\delta_{k}\left(g, d^{\prime}\right)>$ $\beta$

(b) for every $d \in\left(I^{X}\right)^{E}$ either $\delta_{k}\left(f_{n}, d\right)>\beta$ or $\delta_{k}\left(g, d^{\prime}\right)>$ $\beta$.

Indeed, assume that neither (a) nor (b) holds. Then there are $d_{1}, d_{2} \in\left(I^{X}\right)^{E}$ such that $\delta_{k}\left(h, d_{1}\right)<\beta, \delta_{k}\left(f_{n}, d_{2}\right)<\beta$, $\delta_{k}\left(g, d_{1}^{\prime}\right)<\beta, \delta_{k}\left(g, d_{2}^{\prime}\right)<\beta$. Letting $d=d_{1} \sqcap d_{2}$ we get $\delta_{k}(f, d) \vee \delta_{k}\left(g, d^{\prime}\right)<\beta$; however, this contradicts the fact that $(f, g) \in \Sigma_{\beta}^{k}$.

Suppose that (a) holds. Then since $h \sqsubseteq f$ and $\delta_{k}(f, g)<$ $\beta$ we conclude that $\delta_{k}(h, g)<\beta$ and hence $(h, g) \in \Sigma_{\beta}^{k}$. However, this obviously contradicts the assumption of the minimality of the sum $n+l$. In a similar way the case (b) can be excluded. Hence the set $\Sigma_{\beta}^{k}$ is empty.

Thus, $\delta$ is an $(E, K)$-soft fuzzy proximity on $X$. Besides, from its definition it follows that $\delta_{k}(f, g) \leq \delta_{\eta_{\gamma}(k)}^{\gamma}\left(\left(\varphi_{\psi}\right)_{\gamma}(f)\right.$, $\left.\left(\varphi_{\psi}\right)_{\gamma}(g)\right)$ for all $\gamma \in \Gamma$, and therefore all mappings $\left(\varphi_{\psi, \eta}\right)_{\gamma}$ : 
$(X, \delta) \rightarrow\left(X_{\gamma}, \delta^{\gamma}\right)$ are soft proximally continuous. Assume that there exists another $(E, K)$-soft fuzzy proximity $\delta^{*}$ on $X$ such that $\delta^{*} \geq \delta$ and all $\left(\varphi_{\psi, \eta}\right)_{\gamma}:\left(X, \delta^{*}\right) \rightarrow\left(X_{\gamma}, \delta^{\gamma}\right)$ are soft proximally continuous. then there exist $f, g \in$ $\left(I^{X}\right)^{E}$, covers $f=\bigsqcup_{i=1}^{n} f_{i}, g=\bigsqcup_{i=1}^{n} g_{i}$, and $\gamma \in \Gamma$ such that $\delta_{k}^{*}(f, g)>\delta_{\eta_{\gamma}(k)}^{\gamma}\left(\left(\varphi_{\psi}\right)_{\gamma}\left(f_{i}\right),\left(\varphi_{\psi}\right)_{\gamma}\left(g_{j}\right)\right)$ for all $i, j \in\{1, \ldots, n\}$. Since $\delta^{*}$ is an $(E, K)$-soft fuzzy proximity, $\delta_{k}^{*}\left(f_{i_{0}}, g_{j_{0}}\right)=\delta_{k}^{*}(f, g)$ for some $i_{0}, j_{0} \in\{1, \ldots, n\}$ and hence $\delta_{k}^{*}\left(f_{i_{0}}, g_{j_{0}}\right)>\delta_{\eta_{\gamma}(k)}^{\gamma}\left(\left(\varphi_{\psi}\right)_{\gamma}\left(f_{i_{0}}\right),\left(\varphi_{\psi}\right)_{\gamma}\left(g_{j_{0}}\right)\right)$. However, this is impossible because $\left(\varphi_{\psi, \eta}\right)_{\gamma}$ is soft proximally continuous.

Definition 33. By the product of $\left(E_{\gamma}, K_{\gamma}\right)$-soft fuzzy proximity spaces $\left(X_{\gamma}, \delta^{\gamma}\right), \gamma \in \Gamma$, we call the pair $(X, \delta)=\Pi_{\gamma}\left(X_{\gamma}, \delta^{\gamma}\right)$ where $X=\Pi_{\gamma} X_{\gamma}, E=\Pi_{\gamma} E_{\gamma}$ and $K=\Pi_{\gamma} K_{\gamma}$ are the product sets and $\delta$ is the initial $(E, K)$-soft fuzzy proximity for the family of all projections $p_{\gamma}: X \rightarrow X_{\gamma}, q_{\gamma}: E \rightarrow E_{\gamma}$ and $r_{\gamma}: K \rightarrow K_{\gamma}, \gamma \in \Gamma$.

From Theorem 32 it follows that the product $(E, K)$-soft fuzzy proximity $\delta: K \rightarrow I^{\left(I^{X}\right)^{E} \times\left(I^{X}\right)^{E}}$ can be defined by the formula

$$
\begin{gathered}
\delta_{k}(f, g) \\
=\bigwedge\left\{\bigvee_{i, j} \bigwedge_{\gamma} \delta_{r_{\gamma}(k)}^{\gamma}\left(\left(p_{q}\right)_{\gamma}\left(f_{i}\right),\left(p_{q}\right)_{\gamma}\left(g_{j}\right)\right) \mid\right. \\
\left.f=\bigsqcup_{i=1}^{n} f_{i}, g=\bigsqcup_{i=1}^{n} g_{i}, n \in \mathbb{N}\right\} .
\end{gathered}
$$

It is easy to notice that the operation thus defined is indeed the product in the category SFP.

Theorem 34. Let $\left(\varphi_{\psi, \eta}\right)_{\gamma}:(X, \delta) \rightarrow\left(X_{\gamma}, \delta^{\gamma}\right), \gamma \in \Gamma$, be a family of mappings and let $\varphi_{\psi, \eta}:(X, \delta) \rightarrow \Pi_{\gamma}\left(X_{\gamma}, \delta^{\gamma}\right)$ be the diagonal mappings. Then $\operatorname{pd}\left(\varphi_{\psi, \eta}\right)=\bigvee_{\gamma} \operatorname{pd}\left(\left(\varphi_{\psi, \eta}\right)_{\gamma}\right)$.

Proof. Consider the following: $\varphi_{\psi, \eta}:(X, \delta) \rightarrow \Pi_{\gamma}\left(X_{\gamma}, \delta^{\gamma}\right)$, $\left(p_{q, r}\right)_{\gamma}: \Pi_{\gamma}\left(X_{\gamma}, \delta^{\gamma}\right) \rightarrow\left(X_{\gamma}, \delta^{\gamma}\right)$ and $\left(\varphi_{\psi, \eta}\right)_{\gamma}:(X, \delta) \rightarrow$ $\left(X_{\gamma}, \delta^{\gamma}\right), \gamma \in \Gamma$.

Applying Definition 21 and noticing that $\operatorname{pd}\left(\left(p_{q, r}\right)_{\gamma}\right)=0$ we get $\operatorname{pd}\left(\left(\varphi_{\psi, \eta}\right)_{\gamma}\right) \leq \operatorname{pd}\left(\varphi_{\psi, \eta}\right)+\operatorname{pd}\left(\left(p_{q, r}\right)_{\gamma}\right)=\operatorname{pd}\left(\varphi_{\psi, \eta}\right)$ and hence $\bigvee_{\gamma} \operatorname{pd}\left(\left(\varphi_{\psi, \eta}\right)_{\gamma}\right) \leq \operatorname{pd}\left(\varphi_{\psi, \eta}\right)$. To show the converse inequality assume that $\bigvee_{\gamma} \operatorname{pd}\left(\left(\varphi_{\psi, \eta}\right)_{\gamma}\right)<\operatorname{pd}\left(\varphi_{\psi, \eta}\right)$ and find $\varepsilon>$ 0 such that $\sup _{\gamma} \sup _{k \in K} \sup _{f, g \in\left(I^{X}\right)^{E}}\left(\delta_{k}(f, g)-\delta_{\eta_{\gamma}(k)}^{\gamma}\left(\left(\varphi_{\psi}\right)_{\gamma}(f)\right.\right.$, $\left.\left.\left(\varphi_{\psi}\right)_{\gamma}(g)\right)\right)<\left(\sup _{k \in K^{\prime}} \sup _{f, g \in\left(I^{X}\right)^{E}}\left(\delta_{k}(f, g)-\left(\Pi_{\gamma} \delta^{\gamma}\right)_{\eta(k)}\left(\varphi_{\psi}(f)\right.\right.\right.$, $\left.\left.\varphi_{\psi}(g)\right)\right)-\varepsilon$. Choose $f, g \in\left(I^{X}\right)^{E}$ and $k \in K$ realizing the right side of the inequality; then $\delta_{k}\left(f^{*}, g^{*}\right)-\inf _{\gamma} \delta_{\eta_{\gamma}(k)}^{\gamma}\left(\left(\varphi_{\psi}\right)_{\gamma}\left(f^{*}\right),\left(\varphi_{\psi}\right)_{\gamma}\left(g^{*}\right)\right)<\delta_{k}(f, g)-$ $\left(\Pi_{\gamma} \delta^{\gamma}\right)_{\eta(k)}\left(\varphi_{\psi}(f), \varphi_{\psi}(g)\right)-\varepsilon$ for any $f^{*}, g^{*} \in\left(I^{X}\right)^{E}$, and hence $\left(\Pi_{\gamma} \delta^{\gamma}\right)_{\eta(k)}\left(\varphi_{\psi}(f), \varphi_{\psi}(g)\right)<\delta_{k}(f, g)-\delta_{k}\left(f^{*}, g^{*}\right)+$ $\inf _{\gamma} \delta_{\eta_{\gamma}(k)}^{\gamma}\left(\left(\varphi_{\psi}\right)_{\gamma}\left(f^{*}\right),\left(\varphi_{\psi}\right)_{\gamma}\left(g^{*}\right)\right)-\varepsilon$. Consider arbitrary covers $f=\bigsqcup_{i=1}^{n} f_{i}, g=\bigsqcup_{i=1}^{n} g_{i}$ and take $f_{i_{0}}, g_{j_{0}}$ satisfying $\delta_{k}(f, g)=$ $\delta_{k}\left(f_{i_{0}}, g_{j_{0}}\right)$. Then letting $f^{*}=f_{i_{0}}, g^{*}=g_{j_{0}}$ in the above inequality we receive $\beta:=\left(\Pi_{\gamma} \delta^{\gamma}\right)_{\eta(k)}\left(\varphi_{\psi}(f), \varphi_{\psi}(g)\right)+\varepsilon<$ $\inf _{\gamma} \delta_{\eta_{\psi}(k)}^{\gamma}\left(\left(\varphi_{\psi}\right)_{\gamma}\left(f^{*}\right),\left(\varphi_{\psi}\right)_{\gamma}\left(g^{*}\right)\right)$. Thus, for any covers $f=\bigsqcup_{i=1}^{n} f_{i}, g=\bigsqcup_{i=1}^{n} g_{i}$ there exist $i_{0}, j_{0}$ such that $\delta_{\eta_{\gamma}(k)}^{\gamma}\left(\left(\varphi_{\psi}\right)_{\gamma}\left(f_{i_{0}}\right),\left(\varphi_{\psi}\right)_{\gamma}\left(g_{j_{0}}\right)\right)>\beta$ for any $\gamma \in \Gamma$. Noticing that $\varphi_{\psi}(f)=\bigsqcup_{i=1}^{n} \varphi_{\psi}\left(f_{i}\right), \varphi_{\psi}(g)=\bigsqcup_{i=1}^{n} \varphi_{\psi}\left(g_{i}\right)$ and recalling the definition of the product soft fuzzy proximity we conclude that $\left(\Pi_{\gamma} \delta^{\gamma}\right)_{\eta(k)}\left(\varphi_{\psi}(f), \varphi_{\psi}(g)\right) \geq \beta$. The obtained contradiction completes the proof.

Corollary 35. The diagonal $\varphi_{\psi, \eta}:=\Delta_{\gamma}\left(\varphi_{\psi, \eta}\right)_{\gamma}:(X, \delta) \rightarrow$ $\Pi_{\gamma}\left(X_{\gamma}, \delta^{\gamma}\right)$ of a family of mappings $\left(\varphi_{\psi, \eta}\right)_{\gamma}:(X, \delta) \rightarrow$ $\left(X_{\gamma}, \delta^{\gamma}\right), \gamma \in \Gamma$ is soft proximally continuous if and only if each $\left(\varphi_{\psi, \eta}\right)_{\gamma}$ is soft proximally continuous.

Another useful application of initial soft fuzzy proximities is given in the next theorem which describes lattice-theoretic properties of the family of soft fuzzy proximities.

Theorem 36. The set $D$ of all (E, $K)$-soft fuzzy proximities on a given set $X$ is a complete lattice with respect to the order $\leq$.

Proof. Let $\delta^{*}: K \rightarrow I^{\left(I^{X}\right)^{E} \times\left(I^{X}\right)^{E}}$ be defined by for each $k \in K, \delta_{k}^{*}(f, g)=0$ if and only if $f=0_{X}$ or $g=0_{X}$ and $\delta_{k}^{*}(f, g)=1$ otherwise. Obviously $\delta^{*}$ is the largest (i.e., the weakest) $(E, K)$-soft fuzzy proximity on $X$; it will be called antidiscrete. On the other hand let $\delta^{0}: K \rightarrow I^{\left(I^{X}\right)^{E} \times\left(I^{X}\right)^{E}}$ be defined by each $k \in K, \delta_{k}^{0}(f, g)=\sup _{x} \sup _{e \in E}(f \sqcap g)_{e}(x)$. It is also an $(E, K)$-soft fuzzy proximity on $X$. (Indeed, to check up axiom (P5) which is the only unobvious one assume that $\delta_{k}^{0}(f, g)<\inf \left\{\delta_{k}^{0}(f, h) \vee \delta_{k}^{0}\left(h^{\prime}, g\right): h \in\left(I^{X}\right)^{E}\right\}$ for some $f, g \in\left(I^{X}\right)^{E}$. Now taking $h=g$ and noticing that $\delta_{k}^{0}\left(g^{\prime}, g\right)=0$ we obtain a contradiction). Besides, it is easy to notice that $\delta^{0}$ is the strongest (i.e., the smallest) $(E, K)$-soft fuzzy proximity on $X$; it will be called discrete.

Consider any $D_{0} \subset D$ and let $\sigma:=\Pi_{\gamma} \delta^{\gamma}$ be the initial $(E, K)$-soft fuzzy proximity on $X$ for the family of identical mappings $\left(\mathrm{id}_{X \mathrm{id}_{E}, \mathrm{id}_{K}}\right): X \rightarrow(X, \delta), \delta \in D_{0}$. It is easy to notice that $\sigma$ is the greatest lower bound of $D_{0}$ and therefore it will be denoted by inf $D_{0}$. Since $D$ has the largest element $\delta^{*}$, it follows now that $D_{0}$ has the least upper bound $\sup D_{0}$, too.

\section{Conclusion}

All over the globe, (fuzzy) soft set theory is a topic of interest for many authors working in diverse areas due to its rich potential for applications in several directions. So, we found it reasonable to study the fuzzy soft topological structures and also their mutual relationships from the categorical point of view (see $[12,25,28])$. In this study, we considered soft fuzzy proximities introduced in Definition 11, which gives the nearness relation between fuzzy soft sets with respect to the parameters, to be the proximal counterpart of soft fuzzy topologies as they are defined in [12]. We investigate basic properties of these fuzzy proximities and describe how a soft fuzzy proximity generates a soft fuzzy topology. In general topology and also in fuzzy topology, it is important 
to investigate the existence of the initial structures, since the initial structure is the coarsest one which preserves the construction. Based on this fact, we proved the existence of the initial soft fuzzy proximity and by this way we described products in the category SFP.

\section{Conflict of Interests}

The authors declare that there is no conflict of interests regarding the publication of this paper.

\section{References}

[1] D. Molodtsov, "Soft set theory-first results," Computers \& Mathematics with Applications, vol. 37, no. 4-5, pp. 19-31, 1999.

[2] P. K. Maji, R. Biswas, and A. R. Roy, "Fuzzy soft sets”, Journal of Fuzzy Mathematics, vol. 9, no. 3, pp. 589-602, 2001.

[3] H. Aktaş and N. Çağman, "Soft sets and soft groups," Information Sciences, vol. 177, no. 13, pp. 2726-2735, 2007.

[4] A. Aygünoğlu and H. Aygün, "Introduction to fuzzy soft groups," Computers \& Mathematics with Applications, vol. 58, no. 6, pp. 1279-1286, 2009.

[5] F. Feng, Y. B. Jun, and X. Zhao, "Soft semirings," Computers \& Mathematics with Applications, vol. 56, no. 10, pp. 2621-2628, 2008.

[6] Y. B. Jun, "Soft BCK/BCI-algebras," Computers \& Mathematics with Applications, vol. 56, no. 5, pp. 1408-1413, 2008.

[7] Y. B. Jun and C. H. Park, "Applications of soft sets in ideal theory of BCK/BCI-algebras," Information Sciences, vol. 178, no. 11, pp. 2466-2475, 2008.

[8] Y. B. Jun, K. J. Lee, and C. H. Park, "Soft set theory applied to ideals in $d$-algebras," Computers \& Mathematics with Applications, vol. 57, no. 3, pp. 367-378, 2009.

[9] Y. B. Jun, K. J. Lee, and J. Zhan, "Soft $p$-ideals of soft BCIalgebras," Computers \& Mathematics with Applications, vol. 58, no. 10, pp. 2060-2068, 2009.

[10] Q. M. Sun, Z. L. Zhang, and J. Liu, "Soft sets and soft modules," in Proceedings of the 3rd International Conference on Rough Sets and Knowledge Technology (RSKT '08), G. Wang, T. Li, J. W. Grzymala-Busse, D. Miao, A. Skowron, and Y. Yao, Eds., vol. 5009 of Lecture Notes in Computer Science, pp. 403-409, Springer, 2008.

[11] A. P. Šostak, "On a fuzzy topological structure," Rendiconti del Circolo Matematico di Palermo II, no. 11, pp. 89-103, 1985.

[12] A. Aygünoğlu, V. Çetkin, and H. Aygün, "An Introduction to fuzzy soft topological spaces," Hacettepe Journal of Mathematics and Statistics. In press.

[13] A. K. Katsaras, "Fuzzy proximity spaces," Journal of Mathematical Analysis and Applications, vol. 68, no. 1, pp. 100-110, 1979.

[14] A. K. Katsaras, "On fuzzy proximity spaces," Journal of Mathematical Analysis and Applications, vol. 75, no. 2, pp. 571-583, 1980.

[15] G. Artico and R. Moresco, "Fuzzy proximities and totally bounded fuzzy uniformities," Journal of Mathematical Analysis and Applications, vol. 99, no. 2, pp. 320-337, 1984.

[16] G. Artico and R. Moresco, "Fuzzy proximities compatible with Lowen fuzzy uniformities," Fuzzy Sets and Systems, vol. 21, no. 1, pp. 85-98, 1987.

[17] S. A. Markin and A. P. Šostak, "Another approach to the concept of a fuzzy proximity," Rendiconti del Circolo Matematico di Palermo II, no. 29, pp. 529-551, 1992.
[18] Y. C. Kim and K. C. Min, " $L$-fuzzy preproximities and $L$-fuzzy topologies," Information Sciences, vol. 173, no. 1-3, pp. 93-113, 2005.

[19] A. M. Zahran, M. A. Abd-Allah, K. El-Saady, Abd El-Nasser, and G. Abd El-Rahman, "Double fuzzy preproximity spaces," International Journal of Fuzzy Logic and Intelligent Systems, vol. 7, pp. 249-255, 2007.

[20] V. Çetkin and H. Aygün, "Lattice valued double fuzzy preproximity spaces," Computers \& Mathematics with Applications, vol. 60, no. 3, pp. 849-864, 2010.

[21] A. R. Roy and P. K. Maji, "A fuzzy soft set theoretic approach to decision making problems," Journal of Computational and Applied Mathematics, vol. 203, pp. 412-418, 2007.

[22] B. Ahmad and A. Kharal, "On fuzzy soft sets," Advances in Fuzzy Systems, vol. 2009, Article ID 586507, 6 pages, 2009.

[23] A. Kharal and B. Ahmad, "Mappings on fuzzy soft classes," Advances in Fuzzy Systems, vol. 2009, Article ID 407890, 6 pages, 2009.

[24] G. J. Klir and B. Yuan, Fuzzy Sets and Fuzzy Logic Theory and Applications, Prentice Hall PTR, Upper Saddle River, NJ, USA, 1995.

[25] V. Çetkin and H. Aygün, "On soft fuzzy closure and interior operators ," Utilitas Mathematica. In press.

[26] A. Šostak, "On some modifications of fuzzy topology," Matematički Vesnik, vol. 41, no. 1, pp. 51-64, 1989.

[27] B. Tanay and M. B. Kandemir, "Topological structure of fuzzy soft sets," Computers \& Mathematics with Applications, vol. 61, no. 10, pp. 2952-2957, 2011.

[28] V. Çetkin and H. Aygün, “On fuzzy soft topogenous structure," Journal of Intelligent and Fuzzy Systems, 2013. 


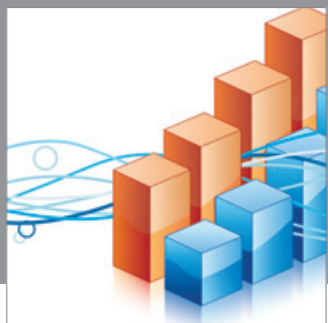

Advances in

Operations Research

mansans

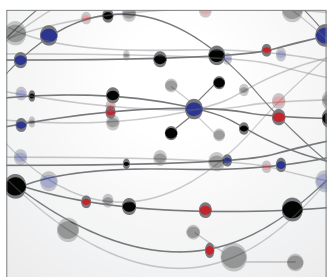

The Scientific World Journal
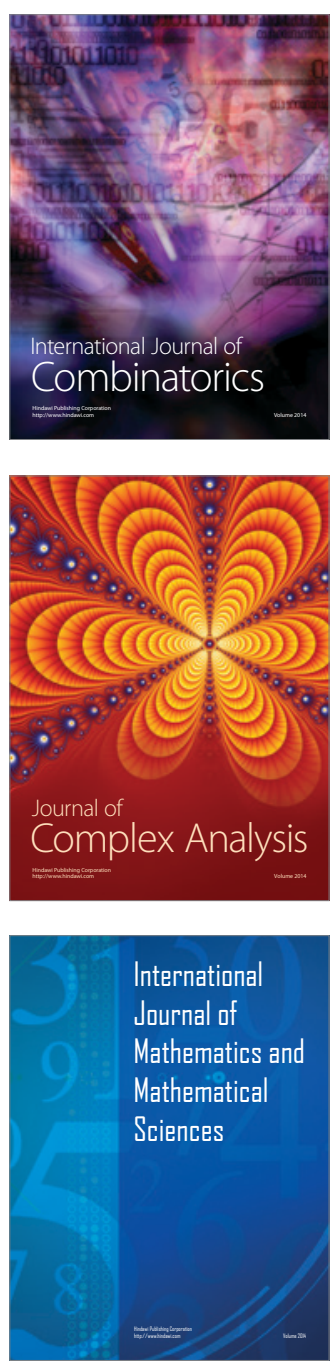
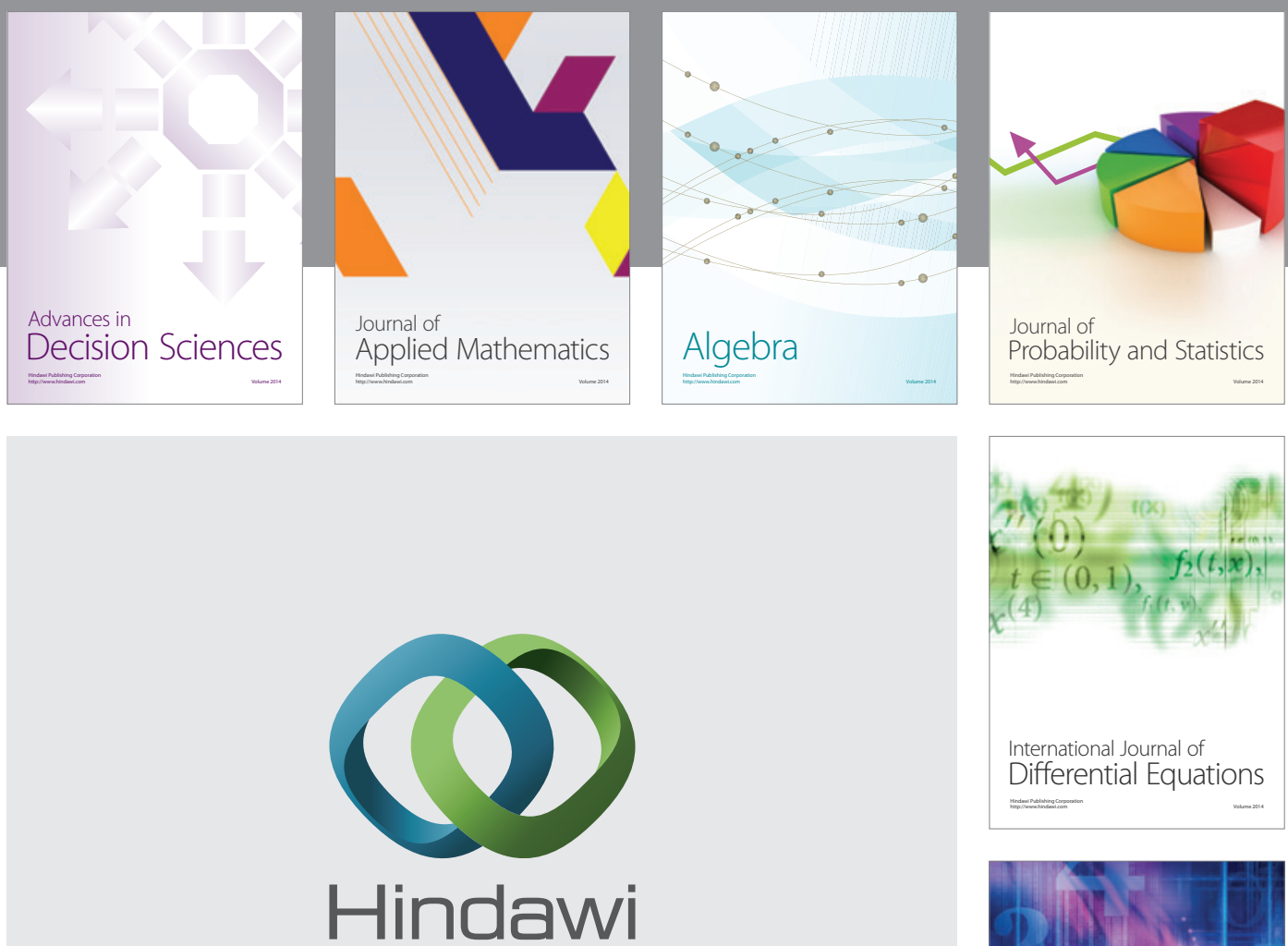

Submit your manuscripts at http://www.hindawi.com
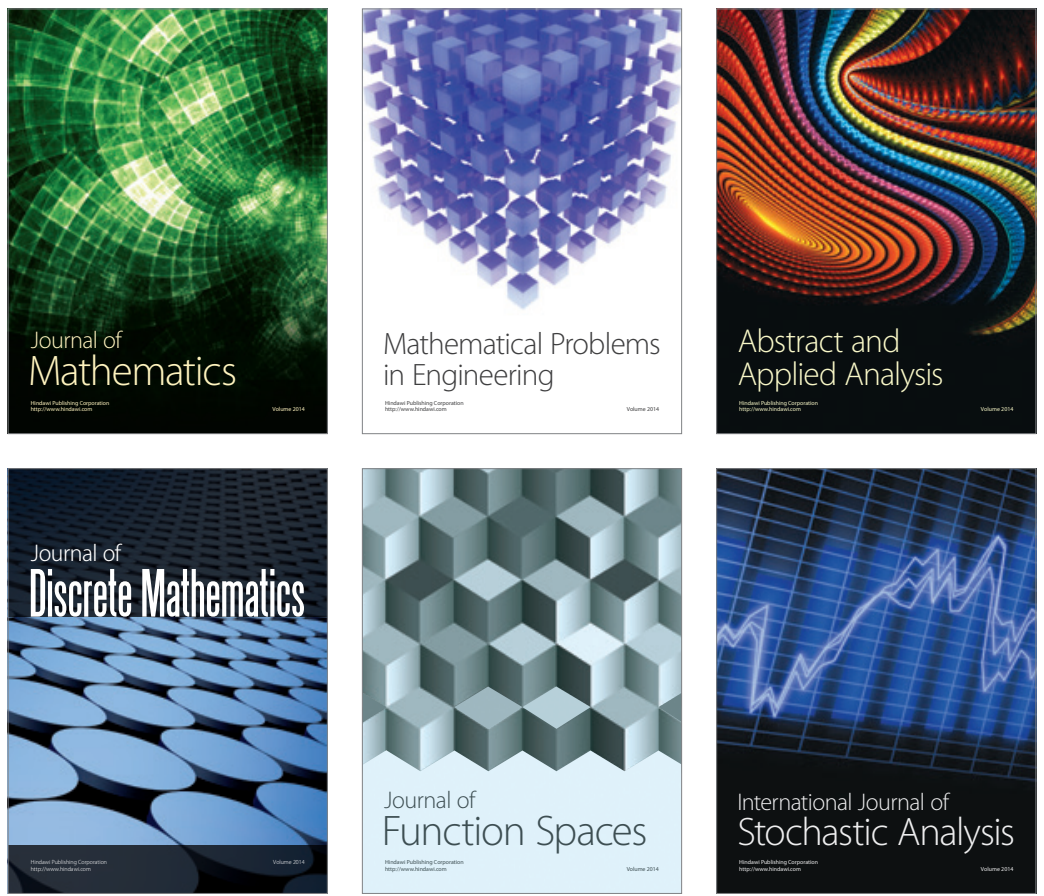

Journal of

Function Spaces

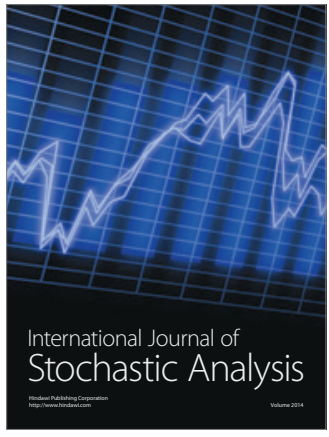

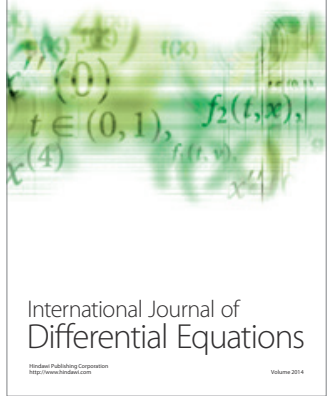
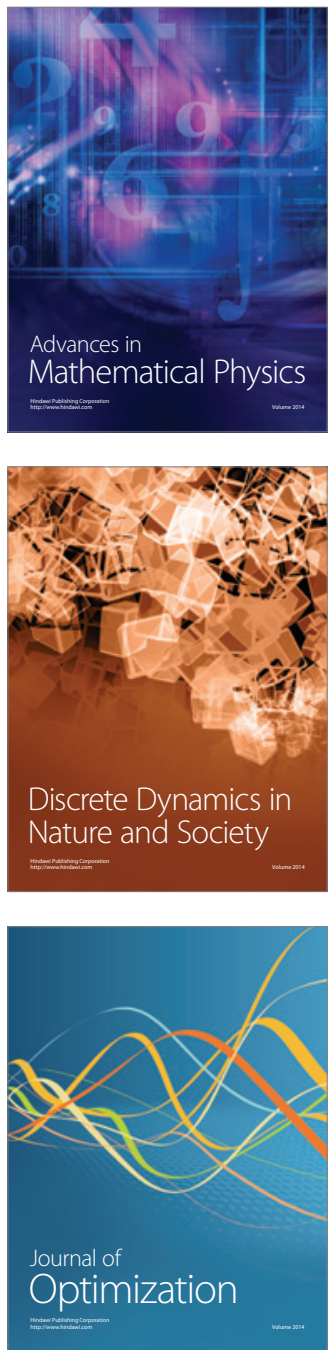